\title{
Management of patient with immune thrombocytopenia with antiphospholipid syndrome and monoclonal gammopathy of undetermined significance
}

\author{
Wiktoria Ryżewska' , Malwina Zarzycka' , Michał Witkowski' \\ Magdalena Witkowska ${ }^{3}$, Tadeusz Robak ${ }^{4}$
}

'Students' Scientific Circle, Department of Hematology, Medical University of Lodz, Poland

${ }^{2}$ Department of Hematology, Copernicus Memorial Hospital, Lodz, Poland ${ }^{3}$ Department of Experimental Hematology, Medical University of Lodz, Poland

${ }^{4}$ Hematology Clinic, Medical University in Lodz, Poland

Correspondence:

Michał Witkowski Department of Hematology, Copernicus Memorial Hospital, Lodz, Poland 93-513 Łódź, ul. Ciołkowskiego 2 phone: +48426895191 e-mail:michalwitkowski13@gmail.com

Received: 30.08.2021 Accepted: 30.10.2021

DOI: 10.24292/01.OR.124301021 Copyright $\odot$ Medical Education. All rights reserved.

\section{ABSTRACT}

Immune thrombocytopenia with antiphospholipid syndrome and monoclonal gammopathy of undetermined significance poses therapeutic dilemmas - whether we should modify the immune thrombocytopenia treatment in antiphospholipid syndrome, what is the influence of monoclonal gammopathy of undetermined significance on the course of immune thrombocytopenia and whether we should and how to prevent the progression of monoclonal gammopathy of undetermined significance to multiple myeloma.

Key words: immune thrombocytopenia, monoclonal gammopathy of undetermined significance, antiphospholipid syndrome 


\section{INTRODUCTION}

Immune thrombocytopenia (ITP) is an acquired blood disease characterized by platelet count $<100 \times 10^{9} /$ I [1]. $80 \%$ of ITP cases is primary and $20 \%$ can be secondary to systemic diseases such as systemic lupus erythematosus (SLE), bone marrow transplantation, viral infections, lymphoproliferative disorders, antiphospholipid syndrome (APS) and other causes $[1,2]$.

APS is a prothrombotic state which is confirmed with the occurrence of $\geq 1$ episodes of arterial or venous thrombosis and the presence of at least one type of antiphospholipid antibodies (aPL) in blood, after 12 weeks. Among the aPL we distinguish lupus anticoagulant (LA), anticardiolipin antibodies $(\mathrm{aCL})$ and anti- $\beta_{2}$ glycoprotein-1 antibodies ( $\left.a \beta_{2} \mathrm{GPI}\right)$ [3]. The diagnosis of antiphospholipid syndrome is tantamount to the diagnosis of high-risk thrombophilia [4].

The concomitance of APS and ITP is therapeutically challenging due to the fact that these are two mutually exclusive disorders. Moreover, ITP alone poses a higher risk of hemorrhage and, paradoxically, thrombosis, due to immunoglobulins-linked activation and consumption of the platelets $[3,5]$.

Consequently, those patients require not only ITP treatment that will raise the platelet count but also anticoagulation to control the excessive blot clot formation. ITP treatment is administered when the platelet count is lower than 20-30 $\times$ $10^{9} / \mathrm{l}$ and the anticoagulative drugs are recommended with thrombocyte count higher that $30-50 \times 10^{9} /$ [ [5]. That causes the dilemma in what manner should we prevent thrombosis in patients with platelet count lower than $30 \times 10^{9} /$.

Another aspect of ITP treatment is the usage of the I line treatment (glucocorticosteroids or intravenous immunoglobulins) which as an adverse effect can cause thrombotic events [5]. In patients with history of thromboembolism, II line treatment should be administered. Among the agents used as an alternative to I line treatment are immunosuppressants such as azathioprine, cyclosporine, cyclophosphamide, vincristine, mycophenolate mofetil, anti-CD20 monoclonal antibody (rituximab), danazol and splenectomy [6].

MGUS (monoclonal gammopathy of undetermined significance, characterized by the presence of monoclonal (M) protein in blood can also possibly elevate the risk of thrombosis in ITP as this abnormal protein can cause an excessive activation of thrombocytes [7]. To diagnose MGUS, undermentioned criteria need to be fulfilled: the amount of M-protein lower than $30 \mathrm{~g} / \mathrm{l}$, exclusion of other B-cell proliferative disorders, no signs of organ impairment and bone marrow clonal plasma cells lower than $10 \%$ in a biopsy. MGUS does not require treatment, however there is a $1 \%$ risk per year of transforming MGUS to multiple myeloma (MM). Therefore, individuals with the presence of paraprotein should be monitored [8].

It is still not verified if ITP can be secondary to MGUS, however the presence of ITP in patients with MGUS is higher than in the general population. The probable reason for that is the paraprotein acting like an autoantibody towards platelets [7]. Herein, we report a case of a patient with ITP and concomitant APS and MGUS.

\section{CASE PRESENTATION}

67-year-old Caucasian male patient was admitted to Haematology Outpatient Clinic due to thrombocytopenia. Patient had a history of deep venous thrombosis (DVT) of right inferior limb. Prior to admission, patient developed DVT of left inferior limb and antithrombotic therapy with low molecular weight heparin (LMWH) - nadroparin was introduced. After 3 days of $L M W H$, patient's platelet count (PLT) decreased from $160 \times 10^{3} / \mu \mathrm{l}$ to $46 \times 10^{3} / \mu \mathrm{l}$ and LMWH was replaced with rivaroxaban in order to exclude heparin induced thrombocytopenia (HIT) as a reason for the drop in patent's PLT. Due to weekend the test for antibodies against heparin-PF4 complex was not obtained.

On admission, patient was asymptomatic with no abnormalities in physical examination. PLT was $53 \times 10^{3} / \mu \mathrm{l}$ with white blood cell (WBC) and red blood cell (RBC) count within the norms. The coagulation profile revealed elevated D-dimers and slightly prolonged prothrombin time (PT). The results of bone marrow biopsy confirmed the diagnosis of ITP. The screening test revealed the presence of $L A$, what in the light of patient's history of DVT, supported the diagnosis of APS. Patient underwent further testing for thrombophilia, where elevated factor VIII (222\%) and homocysteine levels $(16.5 \mu \mathrm{mol} / \mathrm{l})$ were noted. Due to the presence of M-protein in patient's proteinogram $(\mathrm{SMC}=2.8 \mathrm{~g} / \mathrm{l})$, patient was also diagnosed for multiple myeloma (MM). There was no deviations in computed tomography (CT) and the plasmacytes count in bone marrow biopsy was in normal range and no other criteria for MM were confirmed. Thus, patient was established the diagnosis of MGUS IgG. 
During the diagnostic process, patient underwent antithrombotic treatment with rivaroxaban $(20 \mathrm{mg})$, after which he was switched to LMWH (enoxaparin) in the dosage of 1,5 mg per kg once per day due to low and floating PLT level. After 2 months of uneventful therapy, patient's PLT dropped to $20 \times 10^{3} / \mu \mathrm{l}$. LMWH treatment was discontinued. Due to initial phase of DVT and then ITP individualized treatment with azathioprine (50 mg twice per day) and glucocorticosteroids (prednisone $10 \mathrm{mg}$, once per day) was introduced. When PLT was stable, LMWH was applied again but in the lower dose of $1 \mathrm{mg}$ per kg once per day. Further treatment including LMWH, azathioprine and prednisone was continued. When the PLT stabilized above $50 \times 10^{3} / \mu \mathrm{l}$, LMWH was switched to apixaban (first $5 \mathrm{mg}$ twice per day). The decision of resignation from vitamin $\mathrm{K}$ antagonists (VKA) in favour of new oral anticoagulant (NOAC) was established because of patient's non-acceptance of INR measurements. After 6 months, patient was maintained on non-problematic treatment with apixaban in reduced dose (2,5 mg twice per day) and immunosuppressive drugs (azathioprine and prednisone) and PLT was in range $60-80 \times 10^{3} / \mu \mathrm{l}$. The Doppler ultrasonography detected no signs of DVT and control tests were negative.

\section{DISCUSSION}

Sometimes APS is described as a "second hit" for the thrombus formation in ITP [3], however it is still open to debate what factors exactly are responsible for clinical signs of thrombosis. The literature on this subject explains different overlapping mechanisms such as aPL-mediated activation of platelets, of endothelial cells or even impaired fibrinolysis [9]. Among aPL, lupus anticoagulant, detected in above-mentioned patient, is the strongest risk factor for developing thrombosis [10].

Despite these differences, it still remains controversial if patients with concomitance of ITP and APS should be treated differently than patients without aPL antibodies. Conducted studies resulted in conflicted answers. It has been documented that the clinical course of ITP was not different regardless of the presence of aPL antibodies [3]. The response to treatment was alike in both groups of patients [11]. It should also be noted that thrombotic events can occur as a side effect of ITP-specific therapy, thus it is debatable if, in these cases, higher thrombotic risk is associated with aPL or is iatrogenic.

However, some studies show that the outcome of ITP treatment may depend on antiphospholipid antibodies profile [10]. Thus, the therapeutic team needs to find a balance be- tween preventing both thrombosis and hemorrhage. Some patients do not need raising the platelet count; only when it is lower than $20-30 \times 10^{9} / /[12]$.

The choice of antithrombotic drug is another debatable issue in patients with concomitance of ITP and APS. In comparison to VKA, NOACs have wider therapeutic window, fewer drug and dietary interactions and do not require routine monitoring [13]. However, NOACs can be ineffective substituents for VKA in terms of thrombosis prevention in APS patients. There are studies $[14,15]$ reporting that patients treated with rivaroxaban or apixaban have a higher risk of recurrent, mostly arterial, thrombosis compared to patients treated with warfarin. Two main factors associated with recurrent thromboses in patients treated with NOACs have been identified: triple-positive APS and history of arterial thrombosis $[15,16]$.

According to resolutions from $16^{\text {th }}$ International Congress on Antiphospholipid Antibodies [1], consideration of NOAC treatment in APS patients is only reasonable in patients who have single- or double-positive aPL following the first episode of VTE. For triple aPL-positive APS patients it is recommended to switch to VKA.

Moreover, there are differences in treatment profile among drugs in NOAC group. ASTRO-APS study [17] documents that apixaban poses the lowest risk of major bleeding among all NOACs, what could justify the choice of apixaban above other NOACs in treatment of APS patients.

Another question that raises from this case is whether MGUS has an influence on ITP course.

It is known that MGUS is associated with higher thromboembolic risk and occurrence of DVT has been reported in $6,1-7,5 \%$ of patients with MGUS [18]. A retrospective study conducted in 2004, registered that a cumulative DVT rate for MGUS patients was $16 \%$ after 8 years of follow-up [19]. However, the concentration of M-protein is not a very useful predictor of blood coagulation impairment as there are multiple other factors that influence plasma viscosity [20].

The mechanism responsible for higher blood viscosity in monoclonal gammopathies is specific binding of M-protein to platelet receptors and activating them. That results in formation of abnormal clots and consequently with occluding small blood vessels [18]. However, bleeding can occur in patients with paraproteinemia [21] as paraprotein may also bind to coagulation 
factors, which stops their function, or may inhibit fibrin polymerization [22].

Another mechanism involves monoclonal protein showing an intrinsic prothrombotic activity such as lupus anticoagulant-like activity [23]. In that case M-protein binds specifically to phospholipids, components of the cell membrane (fig. 1) [24].
There are ongoing clinical trials on novel drugs for precursor states for MM [28].

Daratumumab is a human monoclonal antibody against CD38 epitope. It has a broad-spectrum killing activity and is currently used in treatment of newly diagnosed and relapsed MM and is in phase II clinical trials, which test its effectiveness in prevention of

Figure 1. The sequence of progression from MGUS to MM. Pathological changes in blood viscosity related to M-protein and risk factors for progressing from MGUS to MM.

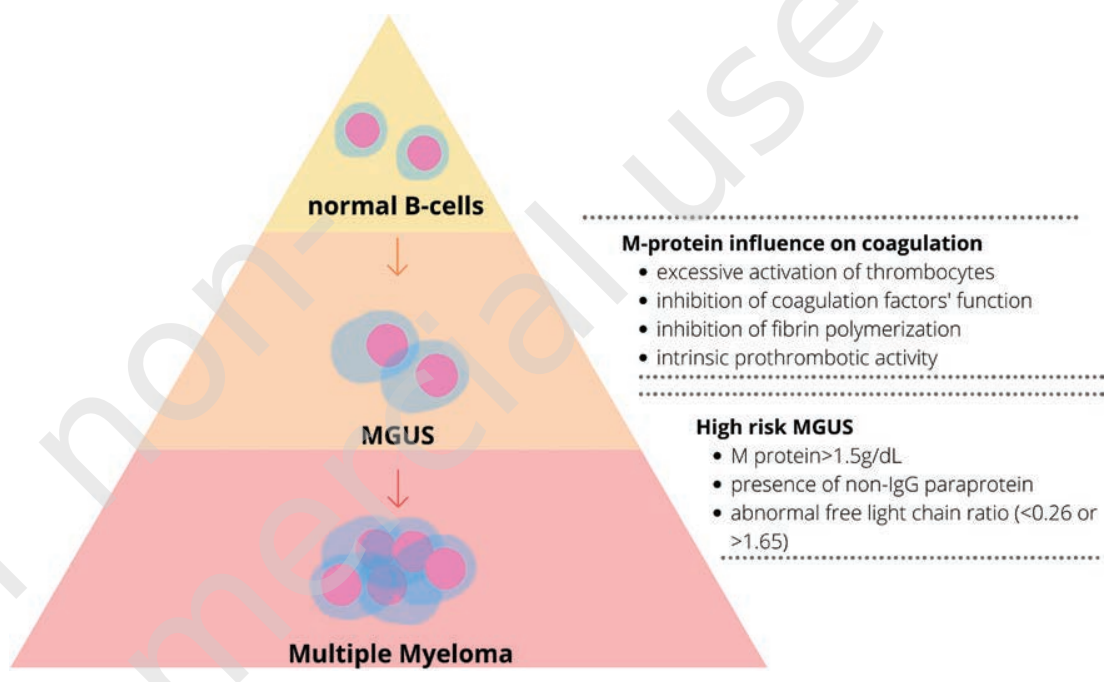

That proves that M-protein can affect coagulation in two contrary manners - enhance both thrombotic and bleeding risk. Thus, it is open to debate and dependent upon patient's condition whether ITP with concomitance of MGUS requires thromboprophylaxis. It has not been confirmed that MGUS exacerbates the course of ITP, however in some studies $[8,25,26]$, it has been proved that the incidence of ITP is higher in MGUS patients than in the general population. Merlini et al. [27] refers that some monoclonal proteins have antigen-binding activity directed to autogenous or foreign antigens. The possible foreign antigens are for example hepatitis $C$ virus or bacterial lipopolysaccharides. According to the study, the antigen-antibody interaction in patients with monoclonal autoimmune syndromes can cause earlier presentation of diseases such as hemolytic anemia or mixed cryoglobulinemia compared to patients who do not have evident antibody activity. However, this mechanism was mainly observed in IgM MGUS and has not been confirmed precisely for MGUS and ITP concomitance.

Although there are currently no recommendations for treatment of MGUS patients, clinical trials have shown that early treatment of MGUS with novel agents could delay its progression to MM. progression from MGUS to MM in patients with high risk MGUS (patients with 3 risk factors identified: M-protein > $1.5 \mathrm{~g} / \mathrm{dl}$, presence of non lgG paraprotein, abnormal free light chain ratio $<0.26$ or $>1.65)$ [29-31].

There is also experimental study in progress, which examines the use of dendritic cell DKK1 vaccine against myeloma. DKK1 is a molecule present on myeloma cells. The vaccine should work by presenting DKK1 to anticancer immune cells via dendritic cells, which should lead to immune attack on myeloma cells [32]. Although, there is a chance of developing drugs which could successfully prevent progression of MGUS to MM, we should take into consideration the fact that these studies have been mainly performed on the high-risk groups of patients only. Moreover, they do not study MGUS-associated morbidity and do not concentrate on minimizing drug-related toxicity [31].

Treating ITP with immunosuppressant also poses a question whether these drugs increase the risk of progression from MGUS to MM. Landgren et al. reported that immunosuppression is one of the risk factors for developing MM [33]. In their cross-sectional analysis, immunosuppression was found in $58 \%$ of patients with 
MGUS progression compared to $20 \%$ of patients without progression. In addition, among patients with MGUS, those with severe immunosuppression, defined as $\geq 2$ suppressed uninvolved immunoglobulins, had significantly higher risk of progression (29\% among patients with disease progression and 3\% among patients without). Moreover, there are also studies presenting MGUS as a cause of secondary antibody deficiency [34], what along with immunosuppressant treatment, could aggravate patient's immunodeficiency. There have also been studies on the use of non-steroidal anti-inflammatory drugs, such as acetylsalicylic acid or celecoxib, in chemoprevention of MM [31].

\section{CONCLUSION}

Considering above mentioned causes, treating ITP with concomitance of APS and MGUS is above all difficult. The final choice of treatment is dependent on a particular patient's clinical picture and therapeutic team's experience with similar cases.

\section{References}

1. Neunert C, Lim W, Crowther M et al. The American Society of Hematology 2011 evidence-based practice guideline for immune thrombocytopenia. Blood. 2011; 117(16): 4190-207. http://doi.org/10.1182/blood-2010-08-302984.

2. Liebman HA. Recognizing and treating secondary immune thrombocytopenic purpura associated with lymphoproliferative disorders. Semin Hematol. 2009; 46: S33-S6. http://doi.org/10.1053/j.seminhematol.2008.12.004.

3. Kim KJ, Baek IW, Yoon $\mathrm{CH}$ et al. Thrombotic risk in patients with immune thrombocytopenia and its association with antiphospholipid antibodies. Br J Haematol. 2013; 161(5): 706-14. http://doi.org/10.1111/bjh.12318.

4. Chayoua W, Kelchtermans H, Moore GW et al. Identification of high thrombotic risk triple-positive antiphospholipid syndrome patients is dependent on anti-cardiolipin and anti-ß2glycoprotein I antibody detection assays. J Thromb Haemost. 2018; 16(10): 2016-23. http://doi.org/10.1111/jth.14261.

5. Vreede AP, Bockenstedt PL, McCune WJ et al. Cryptic conspirators: a conversation about thrombocytopenia and antiphospholipid syndrome. Curr Opin Rheumatol. 2019; 31(3):231-40. http://doi.org/10.1097/BOR.0000000000000595.

6. Witkowski M, Witkowska M, RobakT. Autoimmune thrombocytopenia: Current treatment options in adults with a focus on novel drugs. Eur J Haematol. 2019; 103(6): 531-41. http://doi.org/10.1111/ejh.13319.

7. Rossi D, Paoli LD, Franceschetti $S$ et al. Prevalence and clinical characteristics of immune thrombocytopenic purpura in a cohort of monoclonal gammopathy of uncertain significance. Br J Haematol. 2007; 138(2):249-52. http://doi.org/10.1111/j.1365-2141.2007.06633.x.

8. The International Myeloma Working Group. Criteria for the classification of monoclonal gammopathies, multiple myeloma and related disorders: a report of the International Myeloma Working Group. Br J Haematol. 2003; 121(5): 749-57. http://doi.org/10.1046/j.1365-2141.2003.04355.x.

9. Sikara MP, Grika EP, Vlachoyiannopoulos PG. Pathogenic mechanisms of thrombosis in antiphospholipid syndrome (Aps). In: Tranquilli A ed. Thrombophilia. InTech. 2011. http://doi.org/10.5772/25522.

10. Galli $M$, Luciani $D$, Bertolini $G$ et al. Lupus anticoagulants are stronger risk factors for thrombosis than anticardiolipin antibodies in the antiphospholipid syndrome: a systematic review of the literature. Blood. 2003; 101(5): 1827-32. http://doi.org/10.1182/blood-2002-02-0441.

11. Stasi R, Stipa E, Masi M et al. Prevalence and clinical significance of elevated antiphospholipid antibodies in patients with idiopathic thrombocytopenic purpura. Blood. 1994; 84(12): 4203-8. http://doi.org/10.1182/blood.V84.12.4203.bloodjournal84124203.

12. Neunert C, Terrell DR, Arnold DM et al. American Society of Hematology 2019 guidelines for immune thrombocytopenia. Blood Adv. 2019; 3(23): 3829-66. http://doi.org/10.1182/bloodadvances.2019000966.

13. Arachchillage DJ, Cohen H. Use of new oral anticoagulants in antiphospholipid syndrome. Curr Rheumatol Rep. 2013; 15(6): 331. http://doi. org/10.1007/s11926-013-0331-5.

14. Ghembaza A, Saadoun D. Management of antiphospholipid syndrome. Biomedicines. 2020;8(11): 508. http://doi.org/10.3390/biomedicines8110508.

15. Ordi-Ros J, Sáez-Comet L, Pérez-Conesa M et al. Rivaroxaban versus vitamin k antagonist in antiphospholipid syndrome. Ann Intern Med. 2019; 171(10): 685-94. http://doi.org/10.7326/M19-0291.

16. Dufrost V, Risse J, Reshetnyak $T$ et al. Increased risk of thrombosis in antiphospholipid syndrome patients treated with direct oral anticoagulants. Results from an international patient-level data meta-analysis. Autoimmun Rev. 2018; 17(10): 1011-21. http://doi.org/10.1016/j.autrev.2018.04.009.

17. Woller SC, Stevens SM, Kaplan DA et al. Apixaban for the secondary prevention of thrombosis among patients with antiphospholipid syndrome: study rationale and design (Astro-aps). Clin Appl Thromb Hemost. 2016; 22(3): 239-47. http://doi.org/10.1177/1076029615615960.

18. Zangari M, Elice F, Fink L et al. Hemostatic dysfunction in paraproteinemias and amyloidosis. Semin Thromb Hemost. 2007; 33(4): 339-49. http://doi. org/10.1055/s-2007-976169.

19. Eby C. Pathogenesis and management of bleeding and thrombosis in plasma cell dyscrasias. Br J Haematol. 2009; 145(2): 151-63. http://doi. org/10.1111/j.1365-2141.2008.07577.x.

20. Crawford J, Cox EB, Cohen HJ. Evaluation of hyperviscosity in monoclonal gammopathies. Am J Med. 1985; 79(1): 13-22. http://doi.org/10.1016/00029343(85)90540-6.

21. Djunic I, Elezovic I, Vucic M et al. Specific binding of paraprotein to platelet receptors as a cause of platelet dysfunction in monoclonal gammopathies. Acta Haematol. 2013; 130(2): 101-7. http://doi.org/10.1159/000345418.

22. Robert F, Mignucci M, McCurdy SA et al. Hemostatic abnormalities associated with monoclonal gammopathies. Am J Med Sci. 1993; 306(6): 359-66. http://doi.org/10.1097/00000441-199312000-00001. 
23. Dührsen U, Paar D, Kölbel C et al. Lupus anticoagulant associated syndrome in benign and malignant systemic disease - analysis of ten observations. Klin Wochenschr. 1987; 65(18): 852-9. http://doi.org/10.1007/BF01737004.

24. Ganhão S, Silva B, Rodrigues M et al. P3 Is there a relationship between lupus anticoagulant autoantibody and fatigue in jSLE? In: Poster Presentations. Lupus Foundation of America 2020: A30-A1. http://doi.org/10.1136/lupus-2020-eurolupus.52.

25. Friedriksen H, Schmidt K. The incidence of idiopathic thrombocytopenic purpura in adults increases with age. Blood. 1999; 94(3): 909-13. http://doi. org/10.1182/blood.V94.3.909.415k02_909_913.

26. Neylon AJ, Saunders PWG, Howard MR et al; on behalf of the Northern Region Haematology Group. Clinically significant newly presenting autoimmune thrombocytopenic purpura in adults: a prospective study of a population-based cohort of 245 patients: ITP in Adults. Br J Haematol. 2003 ; 122(6): 966-74. http://doi.org/10.1046/j.1365-2141.2003.04547.x.

27. Merlini G, Stone MJ. Dangerous small B-cell clones. Blood. 2006; 108(8): 2520-30. http://doi.org/10.1182/blood-2006-03-001164.

28. Ho M, Patel A, Goh CY et al. Changing paradigms in diagnosis and treatment of monoclonal gammopathy of undetermined significance (Mgus) and smoldering multiple myeloma (Smm). Leukemia. 2020; 34(12): 3111-25. http://doi.org/10.1038/s41375-020-01051-x.

29. Phipps C, Chen Y, Gopalakrishnan S et al. Daratumumab and its potential in the treatment of multiple myeloma: overview of the preclinical and clinical development. Ther Adv Hematol. 2015; 6(3): 120-7. http://doi.org/10.1177/2040620715572295.

30. Nadeem O, Redd R, Stampleman LV et al. A phase II study of daratumumab in patients with high-risk MGUS and low-risk smoldering multiple myeloma: First report of efficacy and safety. Blood. 2019; 134(suppl 1): 1898. http://doi.org/10.1182/blood-2019-129103.

31. Mouhieddine TH, Weeks LD, Ghobrial IM. Monoclonal gammopathy of undetermined significance. Blood. 2019; 133(23): 2484-94. http://doi. org/10.1182/blood.2019846782.

32. Dendritic Cell DKK1 Vaccine for Monoclonal Gammopathy and Stable or Smoldering Myeloma. Clinicaltrials.gov. https://clinicaltrials.gov/ct2/show/ NCT03591614 (access: 5.06.2021).

33. Landgren O, Hofmann JN, McShane CM et al. Association of immune marker changes with progression of monoclonal gammopathy of undetermined significance to multiple myeloma. JAMA Oncol. 2019; 5(9): 1293. http://doi.org/10.1001/jamaoncol.2019.1568.

34. Lomas OC, Mouhieddine TH, Tahri S et al. Monoclonal gammopathy of undetermined significance (Mgus) - not so asymptomatic after all. Cancers (Basel). 2020; 12(6). http://doi.org/10.3390/cancers12061554.

Conflict of interests:

The authors have disclosed no conflicts of interest.

Financial support:

This research has not received any specific grant from public funding agencies, commercial or non-profit.

Ethics:

The authors had full access to the data and take full responsibility for its integrity.

All authors have read and agreed with the content of the manuscript as written

The paper complies with the Helsinki Declaration, EU Directives and harmonized requirements for biomedical journals. 